\title{
La fissuration d'un sol argileux au cours du des- sèchement. II. Modélisation morphologique
}

\author{
Vincent HALLAIRE \\ I.N.R.A., Station de Science du Sol, Centre de Recherches d'Avignon, F 84140 Montfavet \\ Adresse actuelle: I.N.R.A., Laboratoire de Science du Sol, 65, rue de Saint-Brieuc, F 35042 Rennes.
}

RÉSUMÉ

L'étude des conséquences structurales du retrait d'un sol argileux conduit à proposer un modèle morphologique tridimensionnel. Il permet de calculer les composantes fissurales (largeur et écartement des divers types de fissures) à partir des variables connues (masse et masse volumique des éléments structuraux, épaisseur des couches de sol). Cette analyse confirme que 2 processus se succèdent au cours du dessèchement : la fragmentation et la prise en masse. On décrit ainsi 2 types de réseaux (micro- et macrofissures) qui se distinguent non seulement par leur morphologie, mais également par leur évolution durant le retrait. Enfin, en calculant l'indice des vides pour chaque fraction porale, on propose un bilan de porosité du sol.

Mots clés additionnels : Argile, porosité, fissure, affaissement, densité apparente, mottes, peds, structure du sol.

Study of the structural consequences of the shrinkage of a clayey soil has led us to propose a three-dimensional morphological model. This model computes the width and the spacing of the different types of cracks from known variables, i.e. mass and bulk density of the structural elements, and thickness of soil layers. It accounts quantitatively for the existence of 2 processes that follow one another during drying : splitting up and sintering. We describe 2 kinds of networks (micro- and macrocracks) which differ not only in their morphology, but also in the way in which they develop during shrinkage. Then, by calculation of the void ratio for each kind of cracks, we propose an assessment of soil porosity.

Additional key words : Clay, soil shrinkage, porosity, crack, settlement, bulk density, clods, peds, soil structure.

\section{INTRODUCTION}

Les conséquences du retrait des sols argileux sur leur perméabilité ont incité de nombreux auteurs, depuis STIRK (1954), à relier les caractéristiques hydrodynamiques à la présence des fissures : l'usage de traceurs (BLAKE et al., 1973 ; KISSEL et al., 1973 ; QUISENBERRY \& PHILLIPS, 1976 ; BOUMA et al., 1977) et des mesures de conductivité hydrodynamique (RITCHIE et al., 1972 ; ANDERSON \& BOUMA, 1973 ; MCINTYRE \& SLEEMAN, 1982) ont montré que la percolation à travers les fissures n'obéissait pas aux lois de la diffusion, mais permettait une remontée rapide d'une "nappe profonde " (HOOGMOED \& BOUMA, 1980 ; BOUMA et al., 1980).

Ces travaux soulignent la nécessité d'une connaissance précise de la morphologie des fissures (profondeur, épaisseur et écartement) pour modéliser les conditions de transfert; sur un vertisol, JAILLARD \&
CABIDOCHE (1984) ont ainsi établi d'étroites relations entre l'organisation macroscopique des fissures et la dynamique hydrique du sol.

La description de la fissuration d'un sol argileux (HALlAIRE, 1988), fondée sur une approche structurale - où la disposition des fissures en réseau permet d'étudier les éléments qu'elles individualisent - et effectuée in situ (dessèchement par prélèvement racinaire d'une prairie durant une saison de végétation), nous a conduit à proposer un schéma qualitatif de l'évolution de ce sol au cours du retrait : 2 phases se succèdent, la fragmentation et la prise en masse, occasionnant d'abord un réseau dense de fissures fines, puis un réseau plus lâche de fissures larges.

L'analogie du phénomène mis en évidence avec le comportement de ce même sol à l'affaissement (HALLAIRE, 1987) nous incite à décrire en une seule analyse toutes les caractéristiques fissurales, afin d'en préciser les grandeurs et leurs variations avec l'humidité. Cette 


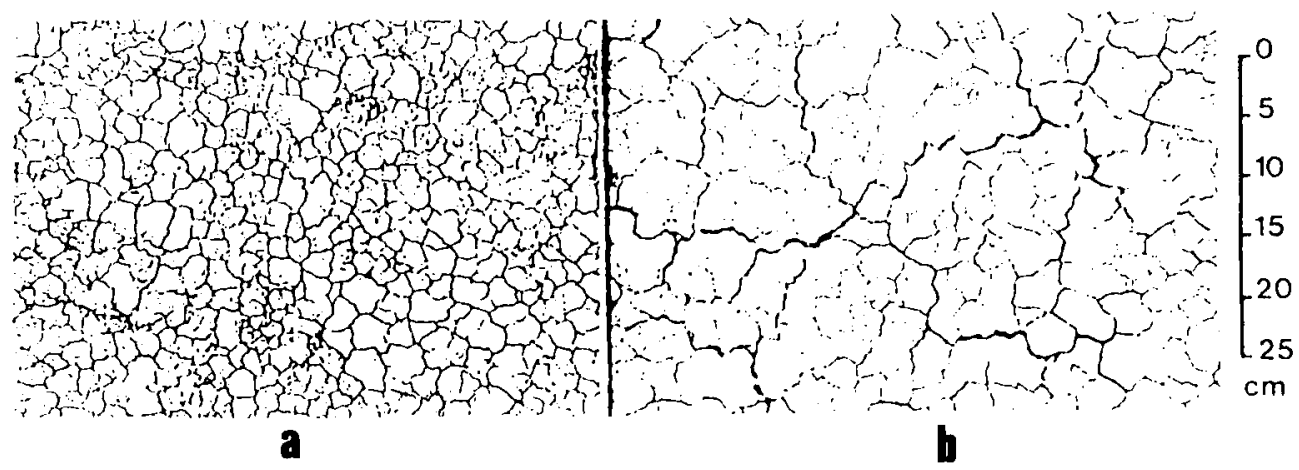

Figure 1

Evolution d'un réseau de fissures entre le début (a) et la fin (b) du dessèchement.

quantification nécessite en premier lieu la formulation d'hypothèses sur l'arrangement géométrique des éléments structuraux et des espaces qui les séparent : cette modélisation morphologique nous permettra alors d'en calculer les diverses composantes.

\section{MÉTHODE D’ANALYSE DU RÉSEAU DE FISSURES}

\section{A. Définition d'un modèle structural du sol}

Dans cette phase d'analyse, les éléments structuraux seront désignés par les termes utilisés dans la phase descriptive : l'élément le plus grossier est la motte, individualisée par un réseau de fissures décelable à l'observation; la motte peut éventuellement se subdiviser en peds, à la faveur de fissures intramottes ou de zones de moindre résistance, non décelables à l'observation mais révélées sous l'action d'une contrainte mécanique imposée à la motte.

Pour représenter l'arrangement des éléments structuraux et des fissures qui les définissent, nous adopterons un modèle morphologique basé sur des observations du sol sur toute la gamme d'humidité. La figure 1 illustre de telles observations : elle représente 2 stades de la fissuration d'une surface nue initialement saturée et desséchée par évaporation à l'air libre ; en $1 a$, le rëseau dense de fissures fines présente son développement maximum; en $1 b$, il a fait place au réseau lâche de grosses fissures, entre lesquelles subsistent encore des fissures très fines en cours de fermeture.

Sans vouloir substituer ce type d'observations essentiellement qualitatives à une analyse d'image plus rigoureuse et basée sur des critères mesurables, nous admettrons en première approximation 2 faits :

- si l'épaisseur des fissures et la taille des éléments structuraux varient au cours du retrait, les caractéristiques de forme de ces éléments structuraux ne paraissent pas varier ;

- les fissures semblent généralement se rejoindre selon des angles proches de $90^{\circ}$; ceci est conforme aux résultats obtenus par HARTGE \& RAHTE (1983) sur des matériaux argileux en cours de retrait.

Ces considérations nous amènent à adopter un modèle morphologique du sol de géométrie cubique.
Development of a network of cracks between the beginning (a) and the end (b) of drying.

Ce modèle s'appuiera sur les hypothèses simplificatrices suivantes (fig. 2) :

- les peds sont assimilés à des cubes d'arête $\lambda_{\mathbf{p}}$. Leur retrait est supposé isotrope ; leur masse volumique sèche $\gamma_{p}$ est supposée varier de façon linéaire avec celle des agrégats, c'est-à-dire de façon inversement proportionnelle avec l'humidité pondérale $\mathbf{w}$;

- les peds d'une même motte sont séparés par des « fissures intramottes ». On distingue des fissures verticales, d'épaisseur $v_{p}$, et des fissures horizontales, d'épaisseur $\zeta_{\mathrm{p}}$;

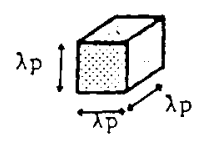

le ped

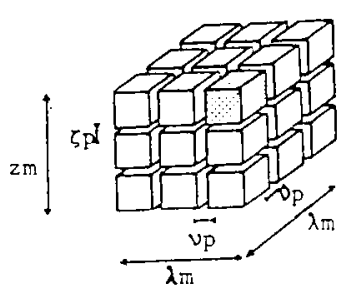

la motte, constituée de $\mathrm{N}$ peds

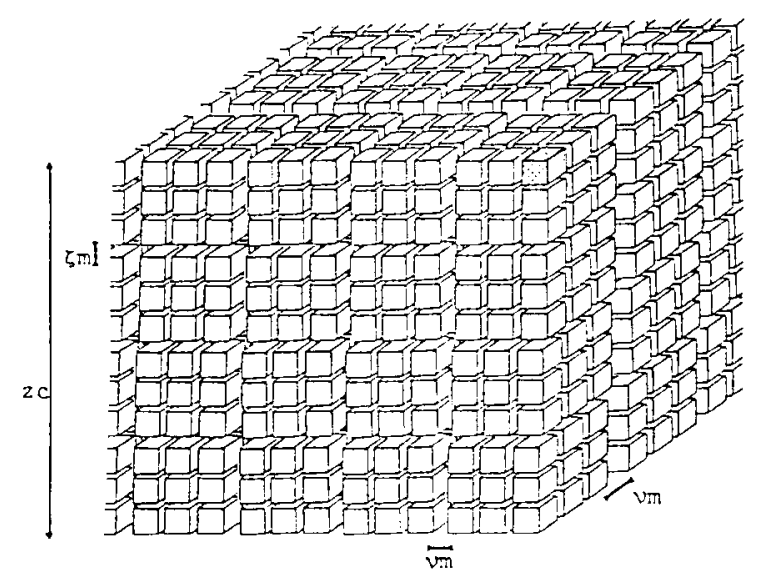

la couche de sol, constituée par l'empilement de N' mottes

Figure 2

Modele morphologique du sol: arrangement des éléments structuraux (mottes et peds) et définition des composantes fissurales.

Morphological model of the soil : arrangement of the structural units (clods and peds) and definition of the components of the cracks. 
- les mottes sont assimilées à des assemblages cubiques de $\mathrm{N}$ peds, c'est-à-dire constituées dans chacune des 3 dimensions par $N^{1 / 3}$ peds. Soit $\lambda_{m}$ leur largeur et $\mathrm{z}_{\mathrm{m}}$ leur hauteur ;

- les mottes d'une même couche sont séparées par des " fissures intermottes ». On distingue ici aussi des fissures verticales, d'épaisseur $v_{m}$, et des fissures horizontales, d'épaisseur $\zeta_{\mathrm{m}}$;

- une couche de sol est constituée par un empilement de $\mathrm{N}^{\prime}$ mottes, séparées par $\left(\mathrm{N}^{\prime}-1\right)$ fissures intermottes horizontales. Elle est caractérisée par sa hauteur réelle, $\mathbf{z}_{\mathfrak{c}}$, et la hauteur cumulée des différents peds qui la composent, $\mathbf{z}_{0}$.

Les hypothèses retenues dans le modèle ont deux conséquences sur la géométrie des fissures, conséquences qui nous permettront de caractériser leur réseau mais qui devront être discutées par la suite :

- les fissures sont toutes assimilées à des lames d'air dont l'épaisseur, pour une humidité donnée, ne varie pas: le cas des étranglements n'est donc pas envisagé ;

- du fait de la géométrie cubique du ped, les fissures se coupent selon des angles droits ; cette orthogonalité des fissures nous permettra de définir l'écartement entre 2 fissures comme étant la taille de l'élément structural qui les sépare. Les valeurs de $\lambda_{p}, \lambda_{m}$ et $\mathbf{z}_{m}$ correspondront donc à l'écartement des fissures intermottes ou intramottes, dans le plan vertical ou dans le plan horizontal.

\section{B. Les éléments de calcul du réseau fissural}

Les méthodes utilisées pour caractériser l'affaissement du sol (HALLAIRE, 1987) et pour décrire sa fissuration au cours du dessèchement (HALLAIRE, 1988) nous permettent d'avoir accès, par la mesure ou le calcul, aux variables suivantes :

w : humidité pondérale du sol en un point donné ;

$\mathbf{z}_{\mathbf{c}}$ : hauteur réelle d'une couche de sol ;

$\mathbf{z}_{0}$ : hauteur calculée d'une couche de sol qui ne comporterait pas de composante horizontale de la fissuration (empilement continu des agrégats) ;

$\mathbf{m}_{\mathbf{m}}$ et $\mathbf{m}_{\mathbf{p}}$ : masses de la motte et du ped ;

$\gamma_{c}, \gamma_{m}$ et $\gamma_{p}$ : masses volumiques sèches de la couche de sol, de la motte et du ped.

A partir de ces variables et du modèle morphologique retenu, on se propose de calculer les caractéristiques structurales $\lambda_{m}$ et $\lambda_{p}, \mathbf{z}_{m}, v_{m}$ et $v_{p}$, et $\zeta_{p}$.

Il n'est cependant pas possible, à ce stade de l'étude, de connaître la répartition de la « composante horizontale de la fissuration » à l'intérieur et à l'extérieur des mottes, c'est-à-dire l'importance relative de $\zeta_{m}$ et de $\zeta_{\mathrm{p}}$ : les seules valeurs qui nous soient accessibles sont le nombre de chacun de ces 2 types de fissures horizontales (intra et intermottes), et leur épaisseur cumulée au sein d'une couche. Bien que cette répartition soit une donnée peu importante pour la caractérisation structurale du sol, et qu'en outre elle évolue probablement au cours du dessèchement, il n'est pas inutile de s'interroger sur l'ordre de grandeur de $\zeta_{\mathrm{m}}$ et de $\zeta_{\mathrm{p}}$. A cette fin, nous envisagerons successivement les 2 cas extrêmes quant à la localisation des fissures horizontales :

- nous supposerons d'abord qu'il n'y a pas de fis- suration horizontale intramotte $\left(\zeta_{p}=0\right)$, mais seulement intermotte. La valeur de $\zeta_{\mathrm{m}}$ déterminée selon cette hypothèse constituera un maximum pour la fourchette dans laquelle se situe l'épaisseur des fissures intermottes ;

- puis, à l'inverse, nous supposerons que la composante horizontale de la fissuration est totalement interne à la motte, mais qu'il n'y a pas de fissuration intermotte $\left(\zeta_{\mathrm{m}}=0\right)$.

Avec les hypothèses géométriques retenues dans le modèle, on peut sans difficulté calculer les composantes du réseau de fissures en fonction des variables connues. On trouvera en annexe les équations utilisées dans les 2 cas extrêmes.

\section{RÉSULTATS}

Les équations précédentes ont permis le calcul des caractéristiques structurales :

- sur les couches supérieures A, B et C (10 à $70 \mathrm{~cm}$ ), dans les 2 cas extrêmes de localisation des fissures horizontales. Les résultats obtenus selon ces 2 hypothèses ne présentent pas de différence significative, ce qui confirme l'importance restreinte que joue cette localisation dans la caractérisation structurale du sol. De ce fait, il ne sera plus tenu compte de cette distinction : seuls seront présentés ici les résultats calculés selon l'hypothèse de fissures horizontales internes aux mottes $\left(\zeta_{\mathrm{m}}=0\right)$;

- sur les couches inférieures $\mathrm{D}$ et $\mathrm{E}(70$ à $110 \mathrm{~cm})$, il n'y a pas de composante horizontale à la fissuration $\left(\zeta_{\mathrm{m}}=\zeta_{\mathrm{p}}=0\right)$, hormis cette différence, les résultats sont rigoureusement semblables à ceux obtenus sur les couches supérieures. Ils ne seront donc pas exposés ici.

\section{A. Evolution de l'épaisseur des fissures}

Les variables $v_{p}, v_{m}$ et $\zeta_{p}$ ont été calculées individuellement pour chaque couple motte-ped, en remplaçant les $\mathbf{z}_{0}, \mathbf{z}_{\mathbf{c}}, \gamma_{\mathbf{c}}, \gamma_{\mathrm{m}}$ et $\gamma_{\mathrm{p}}$ par les moyennes de ces valeurs au sein de chaque classe d'humidité.

Les histogrammes de la figure 3 a présentent, pour chaque classe d'humidité, la distribution des fissures intramottes et intermottes selon leur épaisseur $v_{p}$ et $v_{m}$ : on a distingué 40 classes d'épaisseur entre 0 et $10 \mathrm{~mm}$, chaque classe couvrant une gamme d'épaisseur de $0,25 \mathrm{~mm}$. L'ordonnée représente l'indice des vides correspondant à chaque classe d'épaisseur.

On constate que, quelle que soit l'humidité, l'épaisseur des fissures intramottes reste de l'ordre du demimillimètre. Leur importance diminue notablement jusqu'à 22 p. 100 ; en-dessous de cette teneur en eau, elles représentent un volume très réduit.

L'évolution de l'épaisseur des fissures intermottes présente 2 phases : jusqu'à 23 p. 100 elles forment une population très peu dispersée ne dépassant pas $1 \mathrm{~mm}$ d'épaisseur, donc peu différente de celle des fissures intramottes. A partir de 22 p. 100 , on observe simultanément une augmentation de leur volume, un accroissement global de leur épaisseur - qui atteint en moyenne $5 \mathrm{~mm}$, - et de leur dispersion - qui s'échelonne d'environ 2 à $10 \mathrm{~mm}$. 


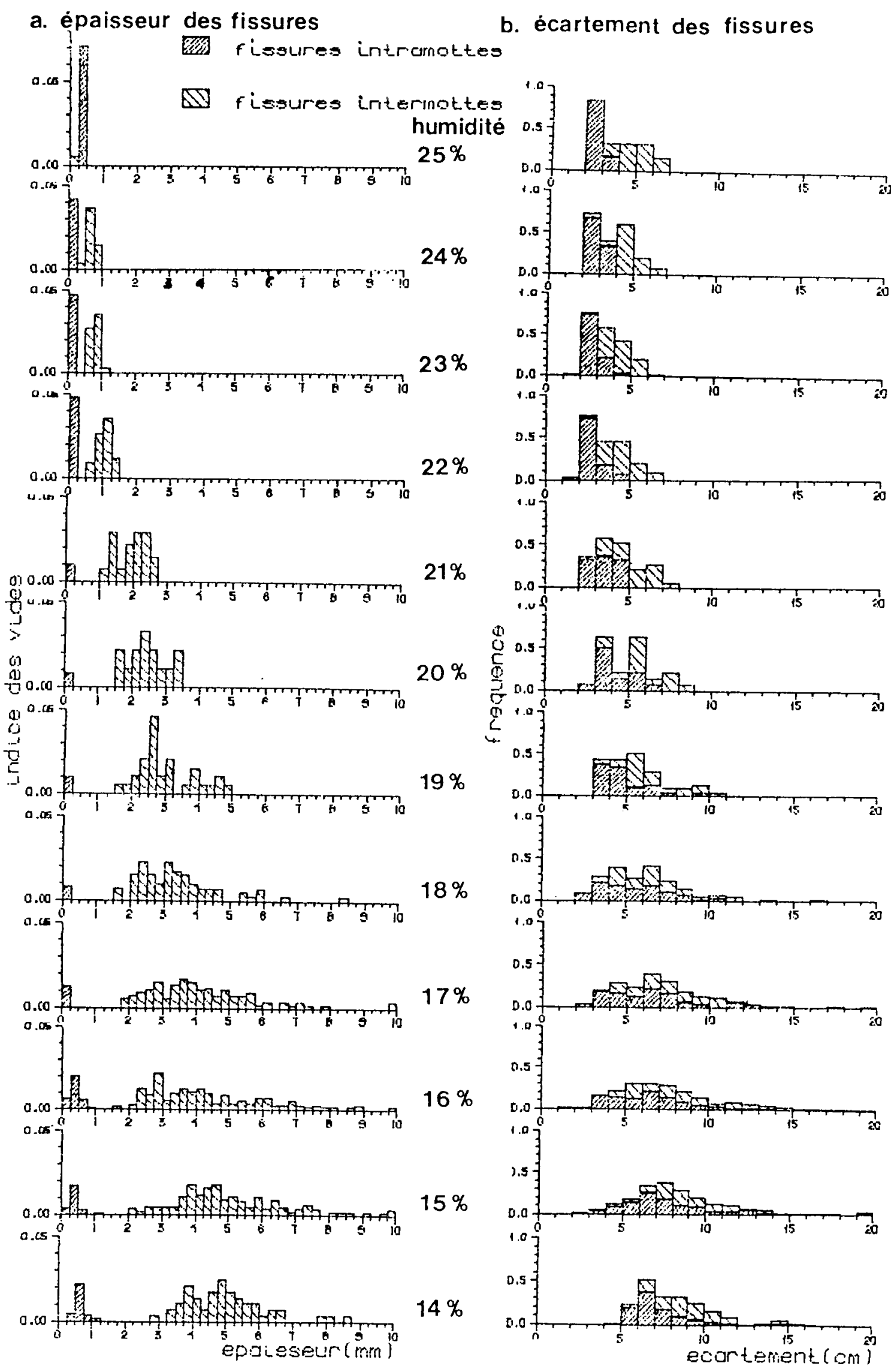

Figure 3

Histogrammes des composantes fissurales intramottes et intermottes pour chaque classe d'humidité. a. épaisseur des fissures ( $\nu_{p}$ et $\left.v_{m}\right)$.

Histograms of the crack components within and outside the clods for each class of moisture. a. crack width $\left(\nu_{p}\right.$ and $\left.\nu_{m}\right) ;$ b. crack spacing $\lambda_{p}$ and $\left.\lambda_{m}\right)$. 
La nature du réseau de fissures évolue donc au cours du retrait : durant la première phase, la faible dispersion d'épaisseur des fissures aboutit à un réseau homogène de fissures fines; durant la seconde phase, la nette différenciation entre fissures intramottes et fissures intermottes, et la dispersion élevée de leur épaisseur aboutissent à un réseau très hétérogène, constitué de fissures larges entre lesquelles peuvent subsister des fissures plus fines.

La figure 4 représente l'évolution moyenne de l'épaisseur des différents types de fissures. Ce schéma fait clairement ressortir la difficulté de distinguer les diverses composantes fissurales dans la première phase de dessèchement ; la seconde phase par contre provoque leur différenciation, par ouverture des unes et fermeture des autres.

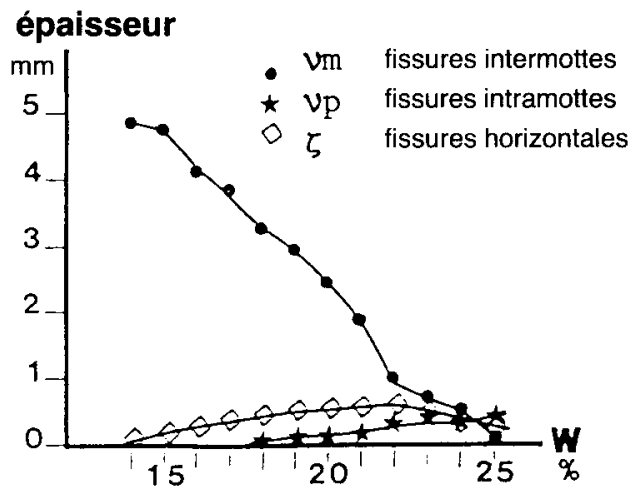

Figure 4

Variation de l'épaisseur moyenne des divers types de fissures avec l'humidité pondérale $w$.

Mean width of the different kinds of cracks versus gravimetric water content $w$.

\section{B. Evolution de l'écartement des fissures}

Nous avons vu plus haut que l'hypothèse d'orthogonalité des fissures retenue dans le modèle morphologique nous autorisait à assimiler la taille des éléments structuraux, $\lambda_{p}$ et $\lambda_{m}$, à l'écartement des fissures verticales qui les séparent.

Les histogrammes de la figure $3 \mathrm{~b}$ présentent, pour chaque classe d'humidité, la distribution de ces variables, en fréquence ; on a distingué 20 classes d'écartement, entre 0 et $20 \mathrm{~cm}$.

Ici encore on peut distinguer 2 phases au cours du dessèchement :

- jusqu'à 23 p. 100 environ, la largeur des peds est peu dispersée et reste de l'ordre de $3 \mathrm{~cm}$; la largeur des mottes, initialement de $5 \mathrm{~cm}$, tend à diminuer dans le même temps pour se rapprocher de celle des peds ;

- à partir de 23 p. 100, la taille des mottes et celle des peds s'accroissent simultanément, pouvant aller jusqu'à $20 \mathrm{~cm}$ de largeur dans les cas extrêmes. La dispersion des valeurs augmente également : en-dessous de 18 p. 100 , cette dispersion est telle qu'il n'est plus possible de distinguer la distribution en taille des mottes et celles des peds. La phase descriptive avait également fait apparaître que, pour ces faibles humidités, la chute de la motte provoquait le détachement mécanique d'éclats plus que la dissociation d'éléments structuraux individualisés.
Ces distributions nous renseignent sur la morphologie du réseau fissural : les fissures sont de plus en plus rapprochées dans la première phase, formant un réseau dense ; elles s'écartent dans la deuxième phase, formant un réseau de plus en plus lâche. Cette évolution en 2 temps est nettement marquée sur la figure 5 , où les valeurs de $\lambda_{\mathrm{m}}$ et $\lambda_{\mathrm{p}}$ sont représentées par leurs moyennes pour chaque classe d'humidité ; pour les teneurs en eau inférieures à 18 p. 100, on a considéré qu'il n'y avait plus de fissures internes aux mottes, celles-ci pouvant être assimilées à des peds.

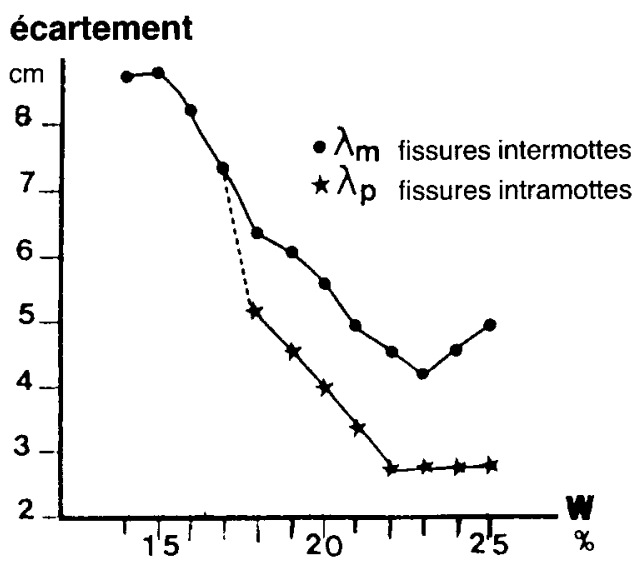

Figure 5

Variation de l'écartement moyen des divers types de fissures avec l'humidité pondérale $w$.

Mean spacing of the different kinds of cracks versus gravimetric water content $w$.

\section{DISCUSSION}

\section{A. Evolution structurale du sol au cours du dessèche- ment}

Les éléments quantitatifs apportés dans cette phase analytique complètent les conclusions proposées dans la phase descriptive, et permettent de préciser les 2 processus mis en évidence : la fragmentation et la prise en masse. Cette évolution est schématisée sur la figure 6 .

La fragmentation opère l'individualisation d'éléments de plus en plus petits, leur dimension minimale étant de l'ordre de $3 \mathrm{~cm}$; cette individualisation résulte de l'ouverture de fissures fines (d'épaisseur moyenne $0,5 \mathrm{~mm}$ ), qui se développent dans les 3 dimensions : une composante horizontale de la fissuration s'oppose ainsi à l'affaissement du sol, dont le retrait vertical est relativement moins important que celui de ses agrégats. La similitude morphologique des fissures internes et externes aux mottes nous amène à qualifier d'homogène le réseau dense de fissures fines résultant de la fragmentation.

La prise en masse intervient lorsque l'humidité du sol passe le seuil de 23 p. 100. Elle opère un regroupement des éléments précédemment individualisés, le retrait s'effectuant alors sur des volumes de plus en plus importants. Ce regroupement provoque une redistribution des espaces fissuraux : les fissures internes aux nouvelles mottes se referment progressivement, les autres fissures continuent de s'ouvrir, mais sont de plus en plus espacées; il en résulte un réseau hétérogène, constitué de fissures larges et espacées entre les- 

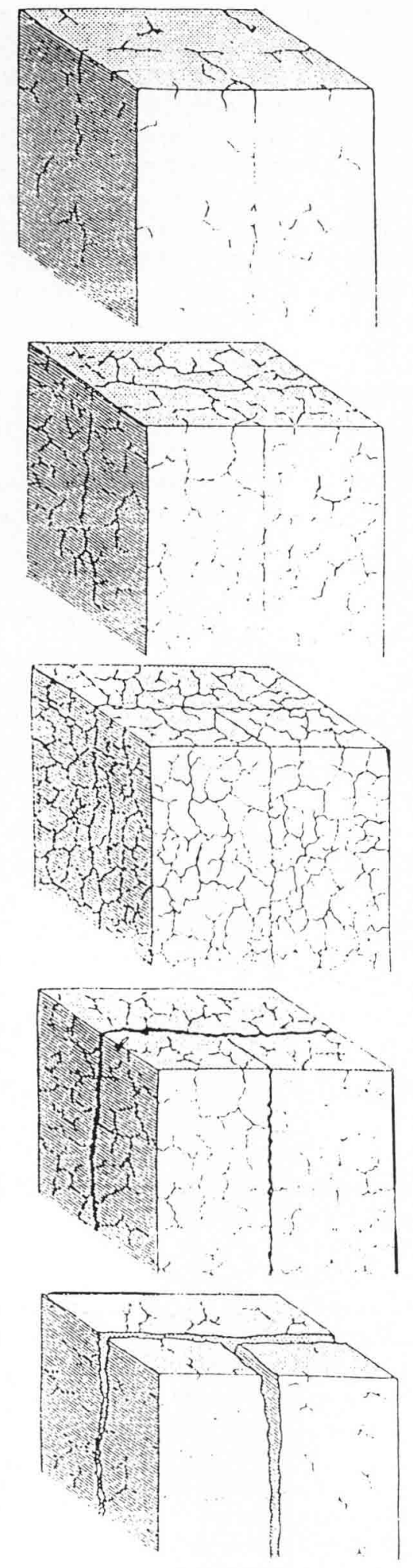

Figure 6

Evolution schématique de la structure du sol au cours du dessèchement.

Schematic representation of the change in soil structure during drying.

quelles se trouvent des fissures fines et serrées. Par ailleurs la composante horizontale de la fissuration tend à s'annuler au cours de cette phase : la gravité tend à rapprocher les éléments structuraux, l'affaissement du sol s'accélère.

\section{B. Bilan poral du sol}

La caractérisation structurale résultant de cette analyse nous permet de distinguer, qualitativement et quantitativement, 2 types de fissures que l'on sait appartenir à 2 types de réseau ; cette distinction ne repose pas seulement sur leur morphologie (épaisseur et écartement) mais également sur leur évolution (ouverture d'un réseau, fermeture d'un autre).

Il est donc parfaitement légitime de subdiviser la porosité fissurale de ce type de sol en différentes catégories, sans que cette distinction revête le caractère arbitraire que prennent parfois les classifications porales basées uniquement sur la taille des pores (micro et macroporosité). Notre discrimination recouvre ici une réalité physique, correspondant à une évolution structurale bien déterminée; l'aspect morphologique n'en est qu'une conséquence.

De ce fait, les fissures externes aux mottes seront désignées par le terme de " macrofissures », quelle que soit leur taille, pour indiquer leur tendance à s'élargir au cours du retrait.

Quant aux fissures internes aux mottes, malgré l'homogénéité de leurs caractéristiques morphologiques (en épaisseur et en écartement) et la similitude de leur évolution (tendance à se fermer au cours du retrait), nous les subdiviserons en 2 ensembles pour tenir compte du rôle différent qu'elles jouent dans le sol (propriétés de transfert, susceptibilité au compactage) :

- le terme de "microfissures » sera réservé à la composante verticale des fissures intramottes ;

- les termes de «vides horizontaux » désigneront leur composante horizontale.

La traduction de ces fractions porales en indice des vides nous permet d'établir un bilan de porosité du sol. La figure 7 représente l'évolution de ce bilan au cours du dessèchement ; il résulte d'une moyenne effectuée sur les couches supérieures. Pour les couches inférieures, la seule différence réside dans l'absence de vides horizontaux.

Ce schéma fait bien ressortir les 2 phases du retrait, séparées par la teneur en eau de 23 p. 100. Il fait égale-

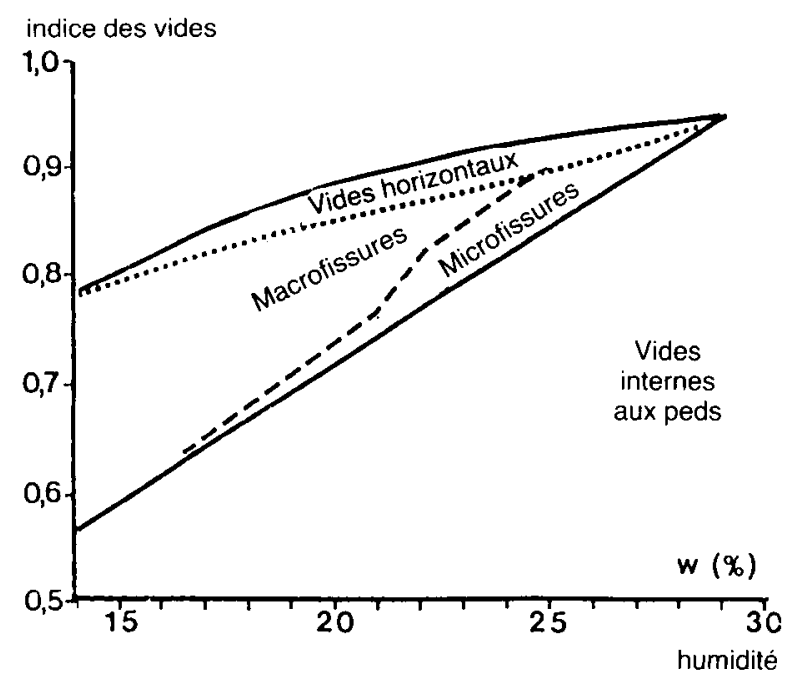

Figure 7

Bilan de porosité du sol : variation de l'indice des vides des diverses fractions porales avec l'humidité pondérale $w$.

Assessment of soil porosity : void ratio of the different kinds of pores versus gravimetric water content $w$. 
ment apparaître que, même si les fissures intermottes existent durant la première phase, l'indice des vides macrofissuraux y est négligeable par rapport aux autres composantes fissurales.

\section{Les limites de l'analyse}

\section{Limites propres au modèle}

L'adoption d'un modèle de géométrie cubique nous a permis d'assimiler les fissures à des lames d'air dont on peut calculer l'épaisseur : des mesures de densité à saturation et des observations aux plus faibles humidités nous avaient en effet montré que les éléments structuraux possédaient des faces, sinon planes, du moins susceptibles de s'emboîter avec un contact parfait ; il n'en reste pas moins vrai que la notion d'épaisseur d'une fissure n'a dans la réalité qu'une signification moyenne, ne reflétant pas sa morphologie complexe (étranglements et élargissements). De plus, le mode de calcul de cette épaisseur simplifie la réalité, puisque cette valeur est obtenue à partir d'un couple motte-ped alors qu'une fissure est définie par 2 mottes (ou peds) contiguës. Enfin, le modèle cubique suppose que les fissures sont perpendiculaires entre elles, ce qui permet d'en calculer un écartement ; or cette notion même d'écartement des fissures perd tout son sens lorsqu'on abandonne l'hypothèse d'orthogonalité.

Il ressort de ces remarques que les résultats issus du modèle ne peuvent être acceptés que sur une population importante, les valeurs calculées prenant alors une signification statistique. Cette justification statistique ne dispense cependant pas de conforter le modèle morphologique proposé : ceci nécessiterait des mesures de sphéricité et d'arrondi des éléments structuraux (au moyen de l'analyse d'image) sur toute la gamme d'humidité ; cela permettrait également de vérifier que les caractéristiques de formes ne varient pas au cours du retrait.

\section{Définition des éléments structuraux}

Les problèmes posés par l'individualisation des mottes et des peds ont été évoqués dans la phase descriptive de cette étude. Au terme de cette phase analytique, ces problèmes apparaissent avec plus d'acuité encore, puisque la distinction structurale en mottes et peds est à la base du modèle, leurs masses intervenant dans tous les termes du calcul.

On a signalé la difficulté de définir la motte lorsque les discontinuités fissurales sont peu visibles, c'est-àdire pour les humidités élevées : la valeur de $\mathbf{m}_{\mathbf{m}}$ est alors assortie d'une grande incertitude. Cependant les résultats précédents ont montré que, pour ces fortes humidités, le volume «macrofissural » était négligeable par rapport à la porosité interne à la motte : le calcul des composantes du réseau sera donc peu affecté par les éventuelles erreurs inhérentes à la mesure de $\mathbf{m}_{\mathbf{m}}$.

La définition du ped à partir de la motte est en ellemême plus objective, puisqu'indépendante de l'observation. Son utilisation dans le modèle ne s'effectue pourtant pas sans risque : le choix du plus gros élément issu de la motte après chute et sa définition comme ped n'étaient initialement destinés qu'à caractériser un état structural interne à la motte, comme indicateur de son degré de fissuration. L'usage de cette donnée dans le modèle risque de surestimer la taille moyenne des éléments structuraux constituant la motte, donc de sousestimer le volume « microfissural ». On peut toutefois considérer que, si un biais affecte les valeurs calculées, il n'est pas susceptible de remettre en cause le processus mis en évidence ni les ordres de grandeur proposés pour les composantes du réseau.

\section{CONCLUSION}

Dans cette seconde partie de l'étude de la fissuration in situ d'un sol argileux, nous nous sommes attaché à analyser, au moyen d'un modèle morphologique, les données obtenues dans la première partie. Non seulement cette analyse a confirmé le processus de fissuration en 2 temps mis en évidence dans la phase descriptive, mais elle a également pu intégrer le processus d'affaissement sur ce même type de sol, en apportant des précisions quantitatives pour chaque étape.

La valeur d'humidité pondérale de 23 p. 100 apparaît ainsi comme un seuil dans le dessèchement, séparant 2 épisodes parfaitement distincts du retrait : la fragmentation et la prise en masse. Si les conséquences structurales d'un tel retrait ont pu être mises clairement en évidence dans les 3 dimensions de l'espace, le mécanisme mis en jeu lors du passage d'une phase à l'autre reste à établir. Sa connaissance serait un élément essentiel pour pouvoir déterminer quels sont les types de sol concernés par un tel phénomène lors de leur retrait.

La méthode utilisée ici constitue donc un outil appréciable pour mettre en évidence une évolution structurale en 2 temps, et pour caractériser quantitativement le réseau fissural tout au long du dessèchement. Le calcul des composantes fissurales, la partition des fissures en plusieurs ensembles, et l'évaluation du volume relatif occupé par chacun d'eux fournissent des valeurs susceptibles d'être utilisées pour la prévision du fonctionnement de ce sol, notamment vis-à-vis des transferts.

Les hypothèses retenues dans le modèle nous amènent cependant à considérer que les résultats numériques n'ont qu'une signification statistique. Un affinement du modèle consisterait à mieux définir les caractéristiques de forme des éléments structuraux (au moyen de l'analyse d'image), et à déterminer plus précisément la taille des peds constitutifs de la motte (au moyen d'une méthode moins grossière que celle utilisée ici).

L'approche proposée ici n'aboutit donc qu'à une caractérisation globale des réseaux de fissures : elle ne saurait se substituer à d'autres méthodes destinées à analyser plus finement la morphologie des pores, ou à chercher l'incidence du contexte cultural sur l'organisation du réseau. Toutefois, en se fondant sur la distinction structurale des éléments de sol isolés par les fissures, cette approche nous a permis de décrire l'évolution de leur réseau et d'évaluer l'importance de leur volume, dans un sol qui se prête mal aux méthodes densimétriques classiques. 


\section{ANNEXE}

Equations d'accès aux composantes fissurales

(Les paramètres calculés sont en caractères gras)

Composante horizontale de la fissuration totalement externe à la motte
Composante horizontale de la fissuration totalement interne à la motte

$$
\zeta_{p}=0
$$

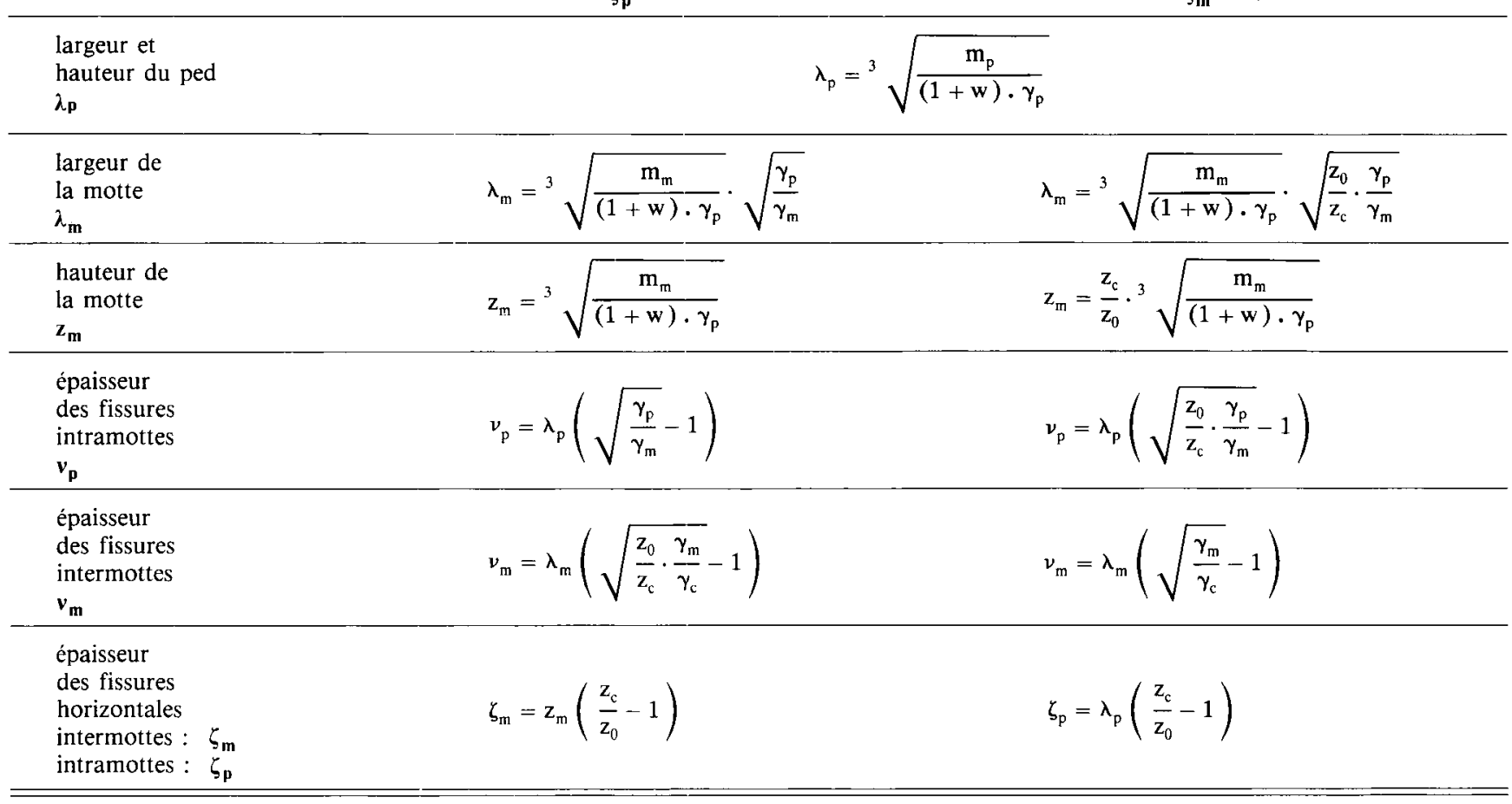

mp : masse du ped

Mm : masse de la motte

$\gamma_{p}:$ masse volumique sèche du ped

$\gamma_{m}$ : masse volumique sèche de la motte

$\gamma_{\mathrm{c}}$ : masse volumique sèche de la couche

$\mathrm{z}_{0}$ : épaisseur cumulée des peds de la couche

$\mathrm{z}_{\mathrm{c}} \quad$ : épaisseur réelle de la couche

w : humidité pondérale de la couche.

\section{RÉFÉRENCES BIBLIOGRAPHIQUES}

Anderson J. L., Bouma J., 1973. Relationships between saturated hydraulic conductivity and morphometric data of an argillic horizon. Soil Sci. Soc. Am. Proc., 37 (3), 408-413.

Blake G., Schlichting E., Zimmermann U., 1973. Water recharge in a soil with shrinkage cracks. Soil Sci. Soc. Am. Proc., 37 (5), 669672.

Bouma J., Jongerius A., Boersma O., Jager A., Schoonderbeek D., 1977. The function of different types of macropores during saturated flow through four swelling soil horizons. Soil Sci. Soc. Am. J., 41 (5), 945-950.

Bouma J., Dekker L. W., Haans J. C. F. M., 1980. Measurement of depth to water table in a heavy clay soil. Soil Sci., 130 (5), 264-270.

Hallaire V., 1987. Retrait vertical d'un sol argileux au cours du dessèchement. Mesures de l'affaissement et conséquences structurales. Agronomie, 7 (8), 631-637.

Hallaire V., 1988. La fissuration d'un sol argileux au cours du dessèchement. 1. Description in situ. Agronomie, 8 (2), 139-145.

Hartge K. H., Rahte I., 1983. Schrumpf- und ScherrisseLabormessungen. Geoderma, 31 (4), 325-336.
Hoogmoed W. B., Bouma J., 1980. A simulation model for predicting infiltration into cracked soil. Soil Sci. Soc. Am. J., 44 (3), 458-461.

Jaillard B., Cabidoche Y. M., 1984. Etude de la dynamique de l'eau dans un sol argileux gonflant : dynamique hydrique. Science $d u$ Sol, 1984 (3), 239-251.

Kissel D. E., Ritchie J. T., Burnett E., 1973. Chloride movement in undisturbed swelling clay soil. Soil Sci. Soc. Am. Proc., 37 (1), 2124.

McIntyre D. S., Sleeman J. R., 1982. Macropores and hydraulic conductivity in a swelling soil. Aust. J. Soil Res., 20 (3), 251-254.

Quisenberry V. L., Phillips R. E., 1976. Percolation of surfaceapplied water in the field. Soil Sci. Soc. Am. J., 40 (4), 484-489.

Ritchie J. T., Kissel D. E., Burnett E., 1972. Water movement in undisturbed swelling clay soil. Soil Sci. Soc. Am. Proc., 36 (6), 874879.

Stirk G. B., 1954. Some aspects of soil shrinkage and the effect of cracking upon water entry into the soil. Aust. J. Agric. Res., 5, 279290. 